\title{
Extreme helium stars: non-LTE matters
}

\section{Helium and hydrogen spectra of the unique objects V652 Her and HD 144941}

\author{
N. Przybilla ${ }^{1}$, K. Butler ${ }^{2}$, U. Heber ${ }^{1}$, and C. S. Jeffery ${ }^{3}$ \\ 1 Dr. Remeis-Sternwarte Bamberg, Sternwartstrasse 7, 96049 Bamberg, Germany \\ e-mail: przybilla@sternwarte.uni-erlangen.de \\ 2 Universitätssternwarte München, Scheinerstrasse 1, 81679 München, Germany \\ 3 Armagh Observatory, College Hill, Armagh BT61 9DG, Northern Ireland
}

Received 22 July 2005 / Accepted 1 October 2005

\section{ABSTRACT}

Quantitative analyses of low-mass hydrogen-deficient (super-)giant stars - so-called extreme helium stars - to date face two major difficulties. First, theory fails to reproduce the observed helium lines in their entirety, wings and line cores. Second, a general mismatch exists for effective temperatures derived from ionization equilibria and from spectral energy distributions. Here, we demonstrate how the issue can be resolved using state-of-the-art non-LTE line-formation for these chemically peculiar objects. Two unique high-gravity B-type objects are discussed in detail, the pulsating variable V652 Her and the metal-poor star HD 144941. In the first case atmospheric parameters from published LTE analyses are largely recovered, in the other a systematic offset is found. Hydrogen abundances are systematically smaller than previously reported, by up to a factor $\sim 2$. Extreme helium stars turn out to be important testbeds for non-LTE model atoms for helium. Improved non-LTE computations show that analyses assuming LTE or based on older non-LTE model atoms can predict equivalent widths, for the He I $10830 \AA$ transition in particular, in error by up to a factor $\sim 3$.

Key words. line: formation - stars: atmospheres - stars: fundamental parameters - stars: individual: V652 Her, HD 144941

\section{Introduction}

Extreme helium stars (EHes) are a rare class of hydrogendeficient objects showing spectral characteristics of early A to late O-type (super-)giants. Their chemical composition is dominated by fusion products from the CNO-cycle and/or the $3 \alpha$-process. Therefore, EHes provide important clues for the study of nuclear astrophysics. Most of the two dozen known EHes could be explained by post-AGB evolution, linking R Cr B stars to Wolf-Rayet type central stars of planetary nebulae, see Heber (1986) and Jeffery (1996) for reviews.

V652 Her and HD 144941 are unique among the class members in several aspects. The carbon, nitrogen and oxygen abundances of both stars are consistent with being processed through the CNO cycle, while all other EHes display $\mathrm{C}$-rich material at the surface, indicating that $3 \alpha$-processing has occurred in addition. Both stars have gravities too large for post-AGB evolution. Saio \& Jeffery (2000) suggest that V652 Her may be the result of a $\mathrm{He}+\mathrm{He}$ white dwarf binary merger. HD 144941 is also outstanding because of its strong metal-deficiency, larger than observed in any other EHe star.

Because of their peculiar chemical composition EHes are important testbeds for stellar atmosphere modelling. Since helium is by far the most abundant element, the He line spectrum can be studied in more detail than in any other type of star, e.g. in HD 144941 all forbidden transitions of He I can be measured. Hydrogen is deficient by a factor of 100 or more and therefore the Balmer lines are very weak, unlike in any other B-type star.

Several LTE spectral analyses encountered two difficulties to be most troublesome: I) synthetic spectra have so far not succeeded in matching the observed helium lines in their entirety, and II) spectroscopic and spectrophotometric temperatures differ systematically. As inadequacies in the basic parameter determination can potentially hamper any further interpretation, the issue needs to be resolved. In the following we investigate the steps that need to be taken to improve the modelling, and the consequences of these for our understanding of EHe stars.

\section{Model calculations}

Our model calculations are carried out in a hybrid approach, as commonly used for analyses of analogous B-type stars with normal chemical composition. First, hydrostatic, planeparallel and line-blanketed (via an opacity sampling technique) LTE model atmospheres are computed with the ATLAS 12 code (Kurucz 1996). Note that we have replaced the photoionization data for He I levels with principal quantum number $n=2$ as used by Kurucz with data from the Opacity Project (Fernley et al. 1987). In particular the cross-sections for the $2 \mathrm{p}^{3} \mathrm{P}^{\circ}$ level 

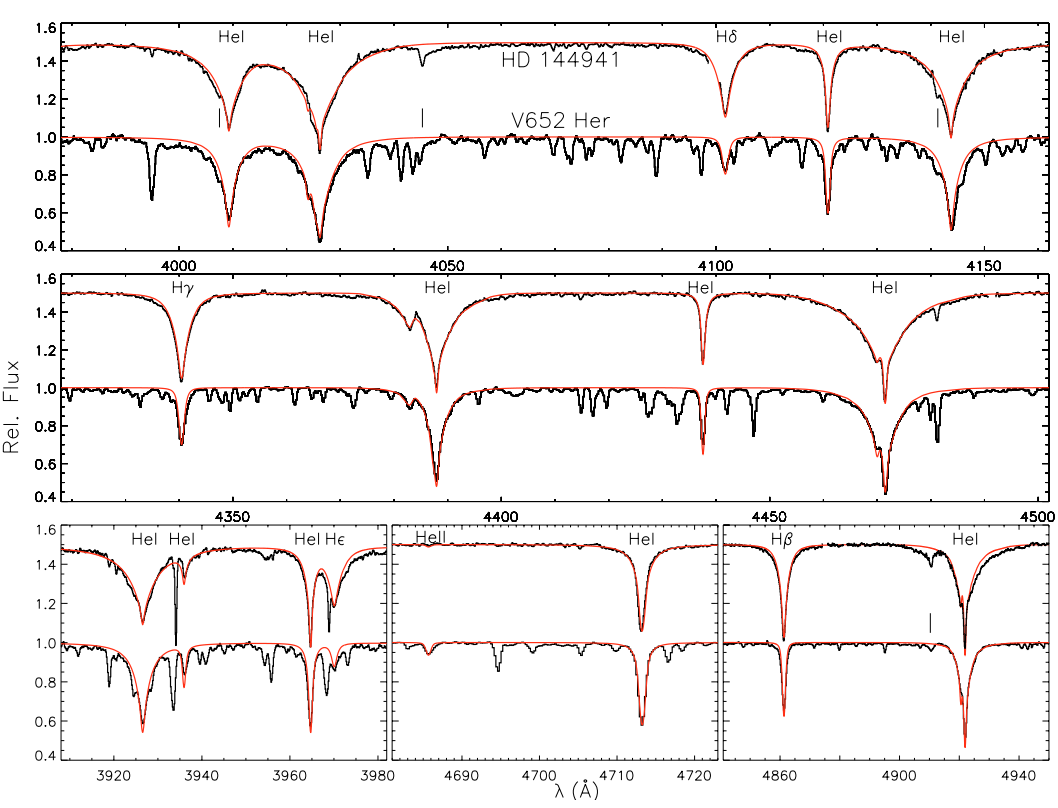

Fig. 1. Comparison of the normalised spectra of V652 Her (lower) and the metal-poor hydrogendeficient star HD 144941 (upper histogram) with our non-LTE spectrum synthesis for helium and hydrogen (full red line): excellent agreement for the entire line profiles - wings and line cores - is found. The He and $\mathrm{H}$ features have been labelled. Forbidden components of He I lines missing in our modelling are indicated by short vertical marks. The red wing of He I $4922 \AA$ in HD 144941 may be affected by an artifact in the data reduction. are increased by a factor $\sim 2$ at threshold, thus improving the fits of computed energy distributions with observation. Stellar parameters and elemental abundances from Jeffery et al. (2001, JWP01) and Harrison \& Jeffery (1997, HJ97) are adopted for the initial models of the stars. Details on the observations can also be found there, which are complemented by further data from Jeffery et al. (1999).

Then, non-LTE line formation is performed on the resulting model stratifications. The coupled radiative transfer and statistical equilibrium equations are solved and spectrum synthesis with refined line-broadening theories is performed using DETAIL and SURFACE (Giddings 1981; Butler \& Giddings 1985). Both codes have undergone major revisions and improvements over the past few years. State-of-theart model atoms for hydrogen (Przybilla \& Butler 2004, PB04) and helium (Przybilla 2005, P05) are utilised, which differ from older model atoms mostly by use of improved collision data from ab-initio computations for electron impact excitation processes. For He I these are the data from Bray et al. (2000) and Sawey \& Berrington (1993), and for H I the data from computations by Butler, as summarised in PB04 - see this work and P05 for further details on the modelling. For comparison, additional calculations are made using the helium model atom of Husfeld et al. (1989, HBHD89). The theory of Barnard et al. (1969) which is discussed further by Auer \& Mihalas (1972) is utilised for a realistic description of line broadening, supplemented by data from Dimitrijević \& Sahal-Bréchot (1990).

The spectrum synthesis is compared to observation in order to derive improved values for the stellar parameters and hydrogen and helium abundances, giving "best fits" in an iterative approach. Effective temperatures $T_{\text {eff }}$ are determined from the He I/II non-LTE ionization equilibrium and the Starkbroadened He I lines act as surface gravity indicators. Stellar parameters of the final models (with estimated uncertainties) are summarised in Table 1, including microturbulence $\xi$. For V652 Her the atmospheric parameters agree very well with those found by JWP01, except for the hydrogen abundance $n_{\mathrm{H}}$,
Table 1. Photospheric parameters of the sample stars.

\begin{tabular}{lcc}
\hline \hline & V652 Her $\left(R_{\max }\right)$ & HD 144941 \\
\hline$T_{\text {eff }}(\mathrm{K})$ & $22000 \pm 500$ & $22000 \pm 1000$ \\
$\log g(\mathrm{cgs})$ & $3.20 \pm 0.10$ & $4.15 \pm 0.10$ \\
$\xi\left(\mathrm{km} \mathrm{s}^{-1}\right)$ & $4 \pm 1$ & $8 \pm 2$ \\
$n_{\mathrm{H}}^{\text {NLTE }}($ by number $)$ & $0.005 \pm 0.0005$ & $0.035 \pm 0.005$ \\
\hline
\end{tabular}

which is reduced by a factor $\sim 2$ (see Sect. 3.2). For HD 144941 , however, the resulting atmospheric parameters differ significantly from previous work of HJ97, implying a reduction in $T_{\text {eff }}$ by $1200 \mathrm{~K}$ and an increase in surface gravity by a factor $\sim 2$. The reduction in the hydrogen abundance is less pronounced in this star.

\section{Testbed for non-LTE spectrum synthesis}

\subsection{Optical helium line spectrum}

As can be seen from Fig. 1 excellent agreement of the nonLTE spectrum synthesis with the observed line profiles of the He features - wings and cores (and forbidden components) - is finally obtained in our approach. Similar agreement of theory with observation in the visual spectral range is obtained using the HBHD89 model atom. The great improvement achieved becomes obvious when comparing Fig. 1 to Fig. 2 of HJ97 and Fig. 5 of JWP01. This resolves one of the most persistent inconsistencies in quantitative analyses of EHes. Only a few predicted forbidden components (Beauchamp \& Wesemael 1998) are missing because the broadening data are unavailable to us.

The He I lines in the visual experience non-LTE strengthening, facilitated by the non-LTE overpopulation of the $n=2$ states relative to the levels of higher principal quantum number, see Fig. 2. There, the runs of non-LTE departure coefficients $b_{i}=n_{i} / n_{i}^{*}$ (the $n_{i}$ and $n_{i}^{*}$ being the non-LTE and LTE populations of level $i$, respectively) and line source functions $S_{l}$ (relative to the Planck function $B_{v}$ ) are displayed. The non-LTE overpopulation occurs because of recombinations to levels of 


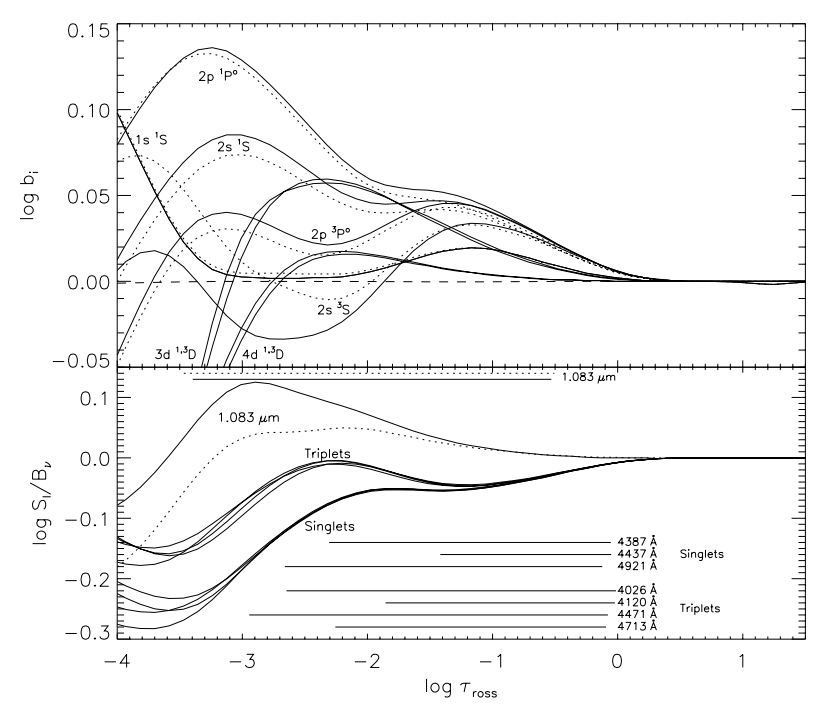

Fig. 2. Departure coefficients $b_{i}$ for He I in the model of V652 Her as a function of Rosseland optical depth $\tau_{\text {ross }}$ for the $n=1$ and 2 and selected $n=3$ and 4 levels from computations using the P05 and the HBHD89 model atoms (upper panel, full and dotted lines, respectively). The He II ground state is also indicated (dashed line). In the lower panel ratios of line source function $S_{l}$ to Planck function $B_{v}$ for several $\mathrm{He}$ I lines are displayed. Line formation depths are indicated. The considerable differences in the populations of the $2 \mathrm{~s}{ }^{3} \mathrm{~S}$ state in the two model atoms translate to a discrepant $S_{l} / B_{v}$ ratio for the $\mathrm{He}$ I $1.083 \mu \mathrm{m}$ transition, giving a substantially weakened line for the modern model atom, while computations based on the old model deviate only little from detailed balance.

He I at higher excitation energies and subsequent de-excitation via downward cascades to the (pseudo-)metastable 2s states (the singlet resonance lines are close to detailed balance). The singlet lines are in general subject to larger non-LTE effects than the triplet lines. Note that the level populations deviate by only a few percent from detailed equilibrium at the formation depths of the continua, showing that the assumption of LTE for the model atmosphere computations is appropriate.

\subsection{Optical hydrogen line spectrum}

In analogy to helium hydrogen can also be supposed to experience non-LTE effects. Departure coefficients for several energy levels and line source-functions for selected $\mathrm{HI}$ lines are displayed in Fig. 3. The H I ground state is strongly depopulated, however at depths irrelevant for the formation of the Lyman continuum. Consequently, non-LTE effects on $\mathrm{H}$ are also unimportant for the atmospheric structure calculations. A small overpopulation of the first excited level occurs at lineformation depths. All other levels of higher $n$ are closely coupled to the continuum (which is in detailed balance) at lineformation depths. This results in a non-LTE strengthening of the Balmer lines, with non-LTE and LTE equivalent widths for e.g. $\mathrm{H} \beta$ differing by more than $30 \%$. A reduction of $\mathrm{H}$ abundances by a factor up to $\sim 2$ is indicated relative to previous LTE studies (for V652 Her the lines are formed on the flat part of the curve of growth).

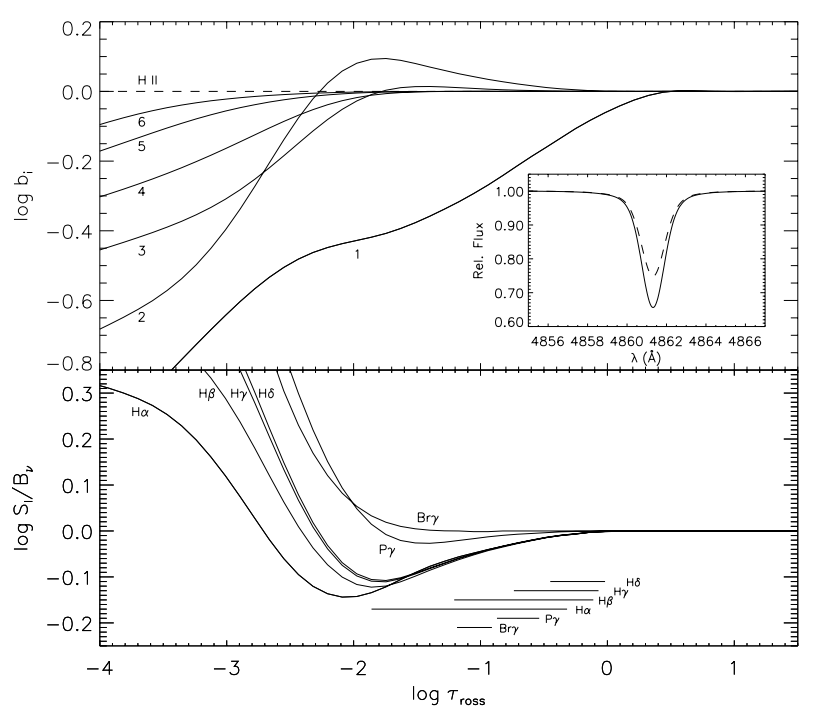

Fig. 3. Same as Fig. 2, but for H I. The $b_{i}$ are labelled by the principal quantum number. Non-LTE (full line) and LTE profiles (dashed line) of $\mathrm{H} \beta$ are compared in the inset. Line source-functions are displayed for Balmer lines and Paschen and Brackett lines in the $J$ - and $K$-band.

\subsection{Infrared line spectra}

Spectral lines in the Rayleigh-Jeans tail of the spectral energy distribution, e.g. in the near-IR for EHes, can experience amplified non-LTE effects (see e.g. PB04). Analyses in the near-IR range are highly useful for constraining the atomic data input for the non-LTE computations, He I in the present case.

Non-LTE weakening is indicated from Fig. 2 for the $2 \mathrm{~s}{ }^{3} \mathrm{~S}-$ $2 \mathrm{p}^{3} \mathrm{P}^{\mathrm{o}}$ transition in the $J$-band, and an emission feature in the case of the $2{ }^{1} \mathrm{~S}-2 \mathrm{p}^{1} \mathrm{P}^{\mathrm{o}}$ transition in the $K$-band. Here, the upper levels are overpopulated relative to the lower states. The He I $1.083 \mu \mathrm{m}$ transition turns out to be highly sensitive to the details of the model calculations, in particular to the use of accurate photoionization cross-sections for the $2 \mathrm{~s}{ }^{3} \mathrm{~S}$ level, and the account of line blocking. As is the case of early-type main sequence stars (P05), large differences to computations based on older He I model atoms such as those of HBHD89 are found. The state-of-the-art model atom predicts the equivalent width $W_{\lambda}$ of He I $\lambda 1.083 \mu \mathrm{m}$ to be a factor $\sim 3$ smaller than the old model atom, which indicates only small deviations from detailed balance.

The significance of the predictions based on the new model atom is demonstrated in Fig. 4, where a comparison is made with the only published spectrum for an EHe star in the near-IR. Good agreement of the model computations with the intermediate-resolution $J$-band observation (JWP01) is found, containing in addition many strong He I lines originating from $n=3$ states. Note however, that the line broadening is dominated by instrumental effects. High-resolution observations would be highly desirable in order to constrain the modelling even further. The analogous $2 \mathrm{~s}{ }^{1} \mathrm{~S}-2 \mathrm{p}{ }^{1} \mathrm{P}^{\mathrm{o}}$ transition in the $K$-band at $2.059 \mu \mathrm{m}$ is predicted to be entirely in emission, with $W_{\lambda} \simeq-200 \mathrm{~m} \AA$ for V652 Her. The non-LTE effects are smaller for HD 144941 because of higher atmospheric densities, implying a lower ionization of the atmospheric helium. Finally, we 


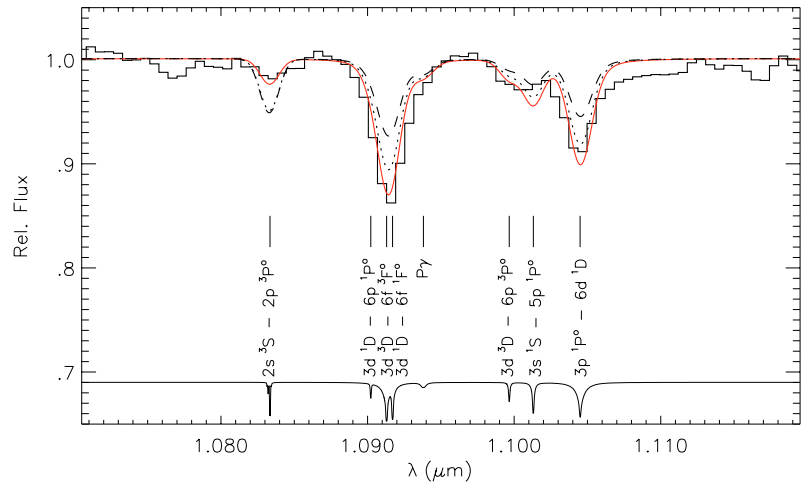

Fig. 4. Comparison of the $J$-band spectrum of V652 Her (histogram) with three model predictions: LTE (dashed line) and two non-LTE computations using the model atom of HBHD89 (dotted line) and the improved model atom of P05 (full red line). In contrast to the other transitions, He I $\lambda 1.083 \mu \mathrm{m}$ experiences strong non-LTE weakening. An unbroadened synthetic spectrum is displayed at the bottom in order to illustrate what can be expected from high-resolution observation.

find that the hydrogen Paschen and Brackett series are much closer to detailed equilibrium than the Balmer lines.

\section{Spectral energy distribution}

The second major difficulty in the modelling of EHes is a general mismatch of effective temperatures derived from ionization equilibria and spectrophotometry. We compare the ATLAS12 model fluxes for stellar parameters derived from the He I/II ionization equilibria (see Table 1) with IUE spectrophotometry and visual and near-IR photometry in Fig. 5. Excellent agreement is found for V652 Her, and reasonable agreement for HD 144941 when interstellar reddening is accounted for.

The fit for HD 144941 may be improved by allowing for changes of the stellar metallicity and/or the effective temperature. Also, a non-standard reddening law cannot be ruled out. A completely satisfactory solution for this metal-poor object is beyond the scope of the present paper, as metal abundances have to be determined, and $T_{\text {eff }}$ should be verified from nonLTE ionization equilibria of metals. However, model atoms for several of the strategic species are unavailable at present.

\section{Conclusions}

The present work demonstrates how a hybrid non-LTE approach using line-blanketed LTE model atmospheres and nonLTE spectrum synthesis succeeds in solving the most persistent problems in the quantitative spectroscopy of EHes. Test computations for two objects imply that stellar parameters from classical analyses of EHes may be subject to systematic modification in the hybrid non-LTE approach. Lower hydrogen abundances than reported previously are found, by up to a factor $\sim 2$.

The atmospheres of the chemically peculiar EHes allow non-LTE model atoms in an environment complementary to normal stars to be tested. The present work verifies the inadequacy of LTE and older non-LTE model atoms for helium. Only a recently improved model atom (P05) reproduces observation for all available spectroscopic indicators, including the

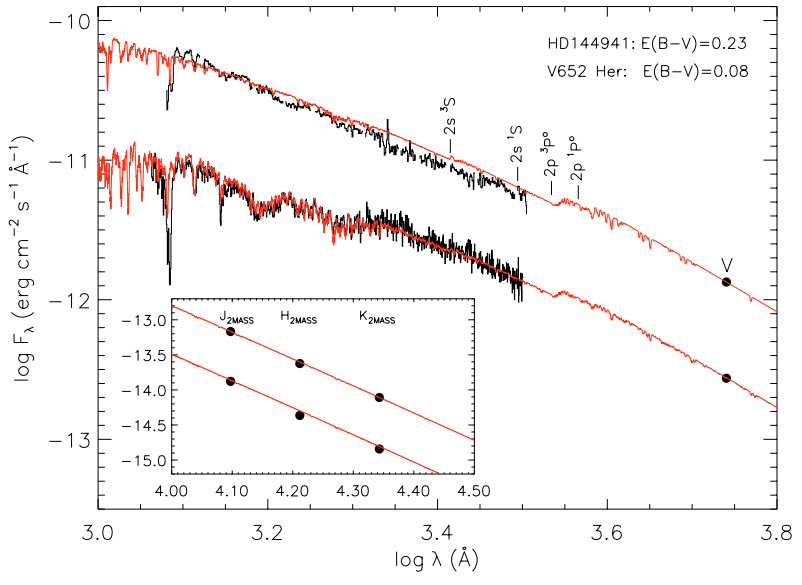

Fig. 5. Fits of theoretical energy distributions (full red lines) to IUE, visual and IR spectrophotometry for V652 Her (near maximum radius, lower jagged curve and dots) and HD 144941 (upper curve). The observed fluxes are dereddened by the amounts indicated and the HD 144941 data are shifted vertically by +0.3 units. The models are normalised to the observed Johnson $V$ magnitudes. Locations for the bound-free edges of the $n=2$ levels of He I are indicated.

He I $\lambda 1.083 \mu \mathrm{m}$ feature in particular, which changes by a factor $\sim 3$ in equivalent width while the lines in the visual remain practically unaffected. High-resolution spectroscopy in the nearUV and near-IR will be required to put even tighter constraints on the atomic model for one of the most basic elements. Finally, an improved understanding of EHes in the context of nuclear astrophysics will require non-LTE studies of metal abundances.

\section{References}

Auer, L. H., \& Mihalas, D. 1972, ApJS, 24, 193

Barnard, A. J., Cooper, J., \& Shamey, L. J. 1969, A\&A, 1, 28

Beauchamp, A., \& Wesemael, F. 1998, ApJ, 496, 395

Bray, I., Burgess, A., Fursa, D. V., \& Tully, J. A. 2000, A\&AS, 146, 481

Butler, K., \& Giddings, J. R. 1985, in Newsletter on Analysis of Astronomical Spectra, No. 9 (London: Univ. London)

Dimitrijević, M. S., \& Sahal-Bréchot, S. 1990, A\&AS, 82, 519

Fernley, J. A., Taylor, K. T., \& Seaton, M. J. 1987, J. Phys. B, 20, 6457

Giddings, J. R. 1981, Ph. D. Thesis, Univ. London

Harrison, P. M., \& Jeffery, C. S. 1997, A\&A, 323, 177 (HJ97)

Heber, U. 1986, in Hydrogen Deficient Stars and Related Objects, ed. K. Hunger, D. Schönberner, \& N. Kameswara Rao (Dordrecht: D. Reidel Publishing Co.), 33

Husfeld, D., Butler, K., Heber, U., \& Drilling, J. S. 1989, A\&A, 222, 150 (HBHD89)

Jeffery, C. S. 1996, in Hydrogen-Deficient Stars, ed. C. S. Jeffery, \& U. Heber (San Francisco: ASP), 152

Jeffery, C. S., Hill, P. W., \& Heber, U. 1999, A\&A, 346, 491

Jeffery, C. S., Woolf, V. M., \& Pollacco, D. L. 2001, A\&A, 376, 497 (JWP01)

Kurucz, R. L. 1996, in Model Atmospheres and Spectrum Synthesis, ed. S. J. Adelman, F. Kupka, \& W. W. Weiss (San Francisco: ASP), 160

Przybilla, N. 2005, A\&A, 443, 293 (P05)

Przybilla, N., \& Butler, K. 2004, ApJ, 609, 1181 (PB04)

Saio, H., \& Jeffery, C. S. 2000, MNRAS, 313, 671

Sawey, P. M. J., \& Berrington, K. A. 1993, At. Data Nucl. Data Tables, 55,81 